\title{
Multi-Location Evaluation of Yield and Yield Related Trait Performance in Bread Wheat Genotypes at Western Oromia, Ethiopia
}

\author{
Geleta Negash", ", Biru Alemu², Wakgari Raga² \\ ${ }^{1}$ Department of Plant Breeding and Genetics, Haro Sabu Agricultural Research Center, Dembi Dollo, Ethiopia \\ ${ }^{2}$ Oromia Agricultural Research Institute (IQQO), Haro Sabu Agricultural Research Center (HSARC), Kellem Wollega, Dembi Dollo, \\ Ethiopia
}

Email address:

geleta2017@gmail.com (G. Negash)

${ }^{*}$ Corresponding author

\section{To cite this article:}

Geleta Negash, Biru Alemu, Wakgari Raga. Multi-Location Evaluation of Yield and Yield Related Trait Performance in Bread Wheat Genotypes at Western Oromia, Ethiopia. International Journal of Applied Agricultural Sciences. Vol. 7, No. 3, 2021, pp. 119-127. doi: $10.11648 /$ j.ijaas.20210703.12

Received: April 5, 2021; Accepted: May 19, 2021; Published: May 31, 2021

\begin{abstract}
Wheat (Triticum aestivum L.) is an important cereal crop, which receives the most attention of specialists in plant breeding and production worldwide. Knowledge of the interaction between genotypes and environment with yield and yield components is a principal aspect of effective selection in crop improvement. Therefore, the objective of this study was: to identify bread wheat genotypes with high level of grain yield and yield stability and insect pest tolerant across locations. The study used 15 bread wheat genotypes, against one local and two standard checks (Liban and Kingbird) at Haro Sabu Agricultural Research Center (HSARC) in 2017-2018 cropping season. Ten agronomic traits and four economically important disease reaction data were evaluated. Analysis of variance detected significant difference, among genotypes in both separated and combined analysis of variance. The combined ANOVA and the additive main effects and multiplicative interactions (AMMI) analysis for grain yield across environments exhibited significantly affected by environments, which explained $65.06 \%$ of the total variation. The genotype and genotype environmental interation were significant and accounted for 13.34 and 9.44\%, respectively. Pricipal component (PCA) 1 and 2 accounted for 7.88 and 1.15\% of the GEI, respectively, with a total of 9.03\% variation. Generally, G6 and G3 were identified as ideal genotypes for yielding ability and stability, tolerant to diseases and use as parents in future breeding programs.
\end{abstract}

Keywords: AMMI, GGEI, Performance, Stability, Triticum aestivum

\section{Introduction}

World wide, wheat (Triticum aestivum L.) is an important cereal crop, which receives the most attention of specialists in plant breeding and production. Yet, its production is limited by the adverse environmental conditions. Environmental fulactuation and interaction with crop plant are the major limitation to wheat production and productivity. Genotype $x$ environment (GE) interaction reduces genetic progress in plant breeding programmes through minimising the association between phenotypic and genotypic values [2]. Therefore, multi-environment yield trials are essential in estimation of genotype by environment interactionn (GEI), identification of superior and stable genotypes in the final selection cycles [11, 12]. Phenotypes are a mixture of genotype $(\mathrm{G})$ and environment (E) components, and their interactions $(\mathrm{G} \times \mathrm{E})$. Genotype by environment interactionn (GEI) complicate process of selecting genotypes with superior performance. Accordingly, Multi-environment trails (METs) are widely used by plant breeders to evaluate the relative performance of genotypes for target environments [4]. The additive main effects and multiplicative interaction (AMMI) model have led to more understanding of the complicated patterns of genotypic responses to the environment [8]. These patterns have been successfully related to biotic and abiotic factors. Yan et al. [20], proposed 
another methodology known as GGE-biplot for graphical display of GE interaction pattern of MET data with many advantages. GGE biplot is an effective method based on principal component analysis (PCA), which fully explores MET data. It allows visual examination of the relationships among the test environments, genotypes and the GE interactions. The first two principle components (PC1 and 2) are used to produce a two dimensional graphical display of genotype by environment interaction (GGE-biplot). If a large portion of the variation is explained by these components, a rank-two matrix, represented by a GGE- biplot, is appropriate [19]. Using a mixed model analysis may offer superior results when the regression of genotype by environment interaction on environment effect does not explain all the interaction [22].

Therefore, the objective of this study was: to identify bread wheat genotypes with high level of grain yield and yield stability and insect pest tolerant across locations.

\section{Materials and Methods}

\subsection{Study sites}

The multi-location yield study (MLYT) was conducted at three locations in Kellem and west Wollega zones of HaroSabu Agricultural Research Center at Belem sub site (altitude 1759 masl, $09^{\circ} 02^{\prime} \mathrm{N}, 035^{\circ} 104^{\prime} \mathrm{E}$ ), Mata (altitude 2016 masl, $08^{\circ} 34^{\prime} \mathrm{N}, 034^{\circ} 44^{\prime} \mathrm{E}$ ) and Badesso (altitude 2054 masl, $08^{\circ}$ $40^{\prime} \mathrm{N}, 034^{\circ} 47^{\prime} \mathrm{E}$ ) in western Oromia, Ethiopia, during the 2017-2018 main cropping season.

\subsection{Breeding materials and experimental design}

A total of 15 genetically diverse bread wheat genotypes (Table1) was evaluated against the checks (Liban, Kingbird and one local check). Arandomised complete block design (RCBD), with three replications, were used. Six rows per plot of $0.2 \mathrm{~m}$ spacing between rows and $2.5 \mathrm{~m}$ row length, and harvestable plot size was $2 \mathrm{~m}^{2}$ (four harvestable rows per plot). A seed rate of $150 \mathrm{kgha}^{-1}$ and fertiliser rate of $100 \mathrm{~kg}$ $\mathrm{ha}^{-1}$ DAP and $150 \mathrm{~kg} \mathrm{ha}^{-1}$ Urea were used.

\subsection{Statistical analysis}

Analysis of variance was calculated using the model:

$$
Y i j=\mu+G i+E j+G E i j
$$

Where:

Yij is the corresponding variable of the $i^{- \text {th }}$ genotype in $j^{- \text {th }}$ environment, $\mu$ is the total mean, Gi is the main effect of $i^{- \text {th }}$ genotype, $\mathrm{Ej}$ is the main effect of $\mathrm{j}^{\text {-th }}$ environment, GEij is the effect of genotype $\mathrm{x}$ environment interaction.

The AMMI model used was:

$$
Y_{i j}=\mu+g_{i}+e_{j}+\sum_{1}^{N} \quad \Lambda k Y_{i k} \delta j k+\varepsilon i j
$$

Where:

Yij is the grain yield of the $\mathrm{i}^{\text {-th }}$ genotype in the $\mathrm{j}^{\text {-th }}$ environment, $\mu$ is the grand mean, gi and ej are the genotype and environment deviation from the grand mean, respectively, $\Lambda \mathrm{k}$ is the eigenvalue of the principal component analysis (PCA) axis k, Yik and $\delta \mathrm{jk}$ are the genotype and environment principal component scores for axis $\mathrm{k}, \mathrm{N}$ is the number of principal components retained in the model, and $\varepsilon_{i j}$ is the residual term.

Table 1. List of bread wheat genotypes evaluated for two years at Western Oromia in Ethiopia.

\begin{tabular}{llll}
\hline No & Codes & Genotypes & Sources \\
\hline 1 & G1 & Local check & Farmer \\
2 & G2 & ETBW7056 & KARC \\
3 & G3 & ETBW7104 & KARC \\
4 & G4 & king bird & KARC \\
5 & G5 & ETBW7068 & KARC \\
6 & G6 & ETBW7076 & KARC \\
7 & G7 & ETBW7077 & KARC \\
8 & G8 & ETBW7072 & KARC \\
9 & G9 & Liban & KARC \\
10 & G10 & ETBW7075 & KARC \\
11 & G11 & ETBW7092 & KARC \\
12 & G12 & ETBW7069 & KARC \\
13 & G13 & ETBW7052 & KARC \\
14 & G14 & ETBW7088 & KARC \\
15 & G15 & ETBW7071 & KARC \\
\hline
\end{tabular}

$\mathrm{G}=$ genotype, ETBW=Ethiopia bread wheat, KARC=Kulumsa Agricultural Reaserch center

GGE-biplot methodology, which is composed of two concepts, the biplot concept [7] and the GGE concept [20] was used to visually analyse the METs data. This methodology uses a biplot to show the factors ( $G$ and $G E)$ that are important in genotype evaluation and that are also the sources of variation in GEI analysis of METs data [18]. The GGE-biplot shows the first two principal components derived from subjecting environment centered yield data (yield variation due to GGE) to singular value decomposition [20].

\subsection{AMMI Stability Value (ASV)}

ASV is the distance from the coordinate point to the origin in a two-dimensional plot of IPCA1 scores against IPCA2 scores in the AMMI model [14]. Because the IPCA1 score contributes more to the GxE interaction sum of squares, a weighted value is needed. This weighted value was calculated for each genotype and each environment according to the relative contribution of IPCA1 to IPCA2 to the interaction sum of squares as follows:

$$
\sqrt{[(\text { SSIPCA1 + SSIPCA2) (IPCA1score })] 2+(\text { IPCA2score }) 2}
$$

Where:

$\mathrm{SS}_{\mathrm{IPCA} 1} / \mathrm{SS}_{\mathrm{IPCA} 2}$ is the weight given to the IPCA1-value by dividing the IPCA1 sum of squares by the IPCA2 sum of squares. The larger the ASV value, either negative or positive, the more specifically adapted a genotype is to certain environments. Smaller ASV values indicate more stable genotypes across environments [14]. 


\subsection{Genotype Selection Index (GSI)}

Stability is not the only parameter for selection as most stable genotypes would not necessarily give the best yield performance. Therefore, based on the rank of mean grain yield of genotypes (RYi) across environments and rank of AMMI stability value RASVi), genotype selection index (GSI) was calculated for each genotype as:

$$
\text { GSIi }=\text { RASVi }+ \text { RYi }
$$

A genotype with the least GSI is considered as the most stable [5]. Analysis of variance was carried out using Statistical Analysis System (SAS) version 9.2 Software [15]. Additive Main Effect and Multiplicative Interaction (AMMI) analysis and GGE bi-plot analysis were performed using Gen Stat $15^{\text {th }}$ edition statistical package [17].

\subsection{Data collection method}

Ten plants were selected randomly before heading from each row (four harvestable rows) and tagged with thread and plant-based data were collected from the sampled plants.

\subsubsection{Plant-based}

Plant height, Spike length, and spike weight, spike lets per spike, grain per spike and grain per spikelet. Plant height $(\mathrm{cm})$; was measured and recorded when reached at $90 \%$ physiological maturity from the ground level to the base of the spike of plant. Spike length $(\mathrm{cm})$; was measured from the base of the spike to the tip of the highest spikelet excluding awns. Spike weight (g); is the average weight of spike of the ten plants randomly selected. Spikelets per spike; is the average number of spikelets of the ten plants randomly selected. Grain per spike; is the average number of grains of the ten plants randomly selected. Grain per spikelet; was calculated by dividing grain per spike by the spikelets per spike.

$$
\text { Grain per spikelet }=\frac{\text { Grain per spike }}{\text { Spikelets per spike }}
$$

\subsubsection{Plot Based}

Days to heading, days to maturity, thousand seed weight, grain yield and four economically important disease reactions like stem rust, leaf rust, septoria and fusarium head blight. Days to heading; was recorded by counting the number of days from sowing to the time when at least $50 \%$ of the heads of the plot fully exerted from the boom or flowered. Days to maturity; was recorded by counting the number of days from sowing to the days when $95 \%$ of the heads of the plot were physiologically matured. Thousand seed weight (g); fife hundred wheat grains were counted and weighed then multiplied by two to obtain thousand seed weight. Grain yield per plot (g); yield per plot was taken and moisture was adjusted to the standard moisture content of $12 \%$ moisture basis after threshing the crop using moisture tester by the following formula. It was calculated as:

\section{Adjusted yield per plot $=$ Actual yield per plot $(100-\mathrm{Y} / 100-\mathrm{X})$}

Where $=$ Actual yield is yield per a given area in a unit at threshing

$\mathrm{Y}=\mathrm{is}$ moisture in $\%$ age at threshing

$\mathrm{X}=\mathrm{is}$ standard moisture in \% age

The disease severity score rated for each pathogen reaction was conducted as per the established procedures for each disease using CIMMYT's method (www.CIMMYT.org).

\begin{tabular}{|c|c|c|c|c|c|c|c|c|}
\hline S. variation & DF & DH & DM & SR & LR & SEP & FHB & PH \\
\hline Rep & 2 & $12.68 * *$ & 12.86 & $0.22 * *$ & 0.03 & 0.02 & $7.37 * *$ & 13.08 \\
\hline Gen & 14 & $265.24 * *$ & $340.90 * *$ & $0.05 *$ & 0.05 & 0.01 & $1.53 * *$ & $578.88 * *$ \\
\hline Loc & 2 & $832.68 * *$ & $5506.27 * *$ & $0.11 *$ & $0.13^{*}$ & 0.03 & $1.30^{*}$ & $7274.29 * *$ \\
\hline $\mathrm{Yr}$ & 1 & $963.33 * *$ & $3998.23 * *$ & 0 & $0.49 * *$ & $0.07 *$ & $76.80 * *$ & $338.08 * *$ \\
\hline Gen.*loc. & 28 & $12.30 * *$ & $47.71 * *$ & 0.02 & 0.03 & 0.01 & 0.15 & $59.27 * *$ \\
\hline Gen. *yr. & 14 & $46.97 * *$ & $122.82 * *$ & 0.02 & 0.05 & 0.01 & $1.37 * *$ & 14.19 \\
\hline Loc. *yr. & 2 & $106.68 * *$ & $3232.78 * *$ & $0.11 *$ & $0.13 *$ & 0.03 & $1.30^{*}$ & $351.89^{* *}$ \\
\hline
\end{tabular}

Table 2. Analysis of variance (ANOVA) for grain yield and yield related traits of bread wheat genotypes evaluated in 2017-2018 main cropping season.

\begin{tabular}{|c|c|c|c|c|c|c|c|}
\hline S. variation & SL & SW & STPS & GPS & GPST & TSW & $\mathrm{Kg} / \mathrm{ha}$ \\
\hline Rep & $3.29 * *$ & 0.41 & $21.25 *$ & $364.69 * *$ & $2.12 * *$ & 198.23 & 42646.84 \\
\hline Gen & $11.37 * *$ & $5.72 * *$ & $35.06 * *$ & $597.59^{* *}$ & $1.31 * *$ & $513.13 *$ & $1831217.12^{* *}$ \\
\hline Loc & $28.99 * *$ & $2.48 * *$ & $293.70 * *$ & $433.04 * *$ & $2.72 * *$ & $2741.55^{* *}$ & $13090998.64 * *$ \\
\hline Yr & $101.14^{* *}$ & $27.60 * *$ & $64.68 * *$ & $3229.91 * *$ & $5.18^{* *}$ & $4066.07 * *$ & $89102882.12 * *$ \\
\hline Gen.*loc. & $0.71 * *$ & 0.25 & $11.54 * *$ & $93.93 *$ & 0.41 & 242.62 & $376887.84 * *$ \\
\hline Gen.*yr. & 0.51 & $0.96 * *$ & 2.99 & 72.64 & 0.31 & 287.65 & 206156.52 \\
\hline Loc.*yr. & $202.90 * *$ & $11.45^{* *}$ & $176.97 * *$ & $4456.30 * *$ & $4.96 * *$ & 261.09 & $4874484.92 * *$ \\
\hline
\end{tabular}

Table 2. Continued.

ns *** non -significant, significant at $5 \%$ and $1 \%$ respectively, Loc *gen=location by genotype, Yr*Loc*gen=year by location by genotype, DF=degree of freedom, DH=Days to Heading, DM=Days to Maturity, $\mathrm{PH}=$ Plant Height; SL=spike Length, SW=Spike Weight, STPS=Spikelets per spike; GPS=-Grain per spike, GPST=Grain per spikelets, TSW=Thousand Seed Weight, YLD kgha ${ }^{-1}=$ Yield in kilogram per hectare 


\section{Results and Discussion}

\subsection{Combined Analysis of Variance (ANOVA)}

Mean square of analysis of variance for all genotypes at different environmental conditions, for grain yield and yield related traits, are presented in Table 2. Highly significant differences were detected among years $(\mathrm{P} \leq 0.01)$ for all parameters, except for stem rust and septoria. The combined analysis of variance revealed that year and location effects were significant for all parameters, except septoria and thousand seed weight. Year*genotypes effects were not significant for all parameters, excluding days to heading, days to maturity, fusarium head blight and spike weight. Year*location * genotypes were significant for some traits such as days to maturity, spike weight, grain per spike and yield. Genotype by environment interaction mean square was highly significant $(\mathrm{P} \leq 0.01)$ for days to maturity, days to heading, plant height, and spike length, spikelets per spike and grain yield.

\subsection{Yield Across Environments}

The performance of the tested bread wheat genotypes for grain yield across location and year are presented in Table 3. Some genotypes (e.g., G6 and G3) are constantly performed best in a group of environments, while other genotypes (such as G14 and G13) are fluctuated across location. The average grain yield ranged from the lowest $\left(3524.47 \mathrm{kgha}^{-1}\right)$ at Belem site in 2017 to the highest $\left(5520.17 \mathrm{kgha}^{-1}\right)$ at Bedesso site in 2018 , with grand mean of $4479.47 \mathrm{kgha}^{-1}$ (Table 3) The grain yield across environments ranged from the lowest of 3925 $\mathrm{kgha}^{-1}$ for local check to the highest of $5069 \mathrm{kgha}^{-1}$ for genotype (G6) (Table3). This wide variation might be due to their genetic potential of the genotypes. Genotype (G6) was the top-ranking pipeline in all environments, except at Belem in 2018. Similarly, genotype (G3) ranked first at all sites, except at Bedeso in 2017 and 2018 cropping season. However, genotype (G8) ranked the least in all environmental sites throughout cropping season (Table3). The difference in yield rank of genotypes across the environments exhibited the high crossover type of genotypes $\mathrm{x}$ environmental interaction [21].

\subsection{Agronomic Performance}

Combined mean grain yield and other agronomic traits are presented in Table 4. High mean of spike length, spike weight, spikelets per spike, grain per spike, grain per spikelets, thousand seed weight and grain yield and medium days to heading and days to maturity were recorded by genotypes (G6). These offer great flexibility for developing improved varieties suitable for various agro-ecologies with variable length of growing period and high in grain yield status. However, G2, G10 and G14 were with short mean of days to heading and days to physiological maturity, indicating that early maturing genotypes were desirable when moisture was the limiting factors of production. Similarly, the local check was recorded with high plant height, indicating that the variety might be susceptible to lodging. Genotypes (G6) and (G3) were with medium plant height indicated, and the possibility for developing resistant varieties against lodging problems. Moreover, genotypes (G6, G3 and G2) recorded the highest grain yield and had $21.3,10.9$ and $4.4 \%$ yield advantages over the best standard check (Liban), respectively (Table 4).

Table 3. Mean grain yield $\left(\mathrm{kgha}^{-1}\right)$ of bread wheat genotypes evaluated at three environments.

\begin{tabular}{|c|c|c|c|c|c|c|c|}
\hline \multirow{3}{*}{ Genotypes } & \multicolumn{7}{|c|}{ Grain yield $\left(\mathrm{kgha}^{-1}\right)$} \\
\hline & \multicolumn{3}{|l|}{2017} & \multicolumn{3}{|l|}{2018} & \multirow{2}{*}{ Com. mean } \\
\hline & Belem & Bedesso & Mata & Belem & Bedesso & Mata & \\
\hline ETBW7052 & $3426.5 \mathrm{c}$ & $3780.6 \mathrm{~cd}$ & $3894.8 d$ & $4477.5 c-f$ & $5394.6 \mathrm{~d}$ & $4707.4 \mathrm{~cd}$ & $4280 \mathrm{ef}$ \\
\hline ETBW7056 & $4057.2 \mathrm{a}$ & $4896 a$ & $4744.4 \mathrm{ab}$ & $3830 \mathrm{f}$ & $6348.5 a$ & $5387.8 \mathrm{ab}$ & $4877 \mathrm{c}$ \\
\hline ETBW7068 & $3531.3 \mathrm{bc}$ & $3689.5 \mathrm{~cd}$ & $4634.2 \mathrm{abc}$ & $4790.3 a-d$ & $5459.4 \mathrm{~cd}$ & $5308.6 \mathrm{ab}$ & $4568 \mathrm{~cd}$ \\
\hline ETBW7069 & $3278.1 \mathrm{~cd}$ & $3395.1 \mathrm{~d}$ & $3841.1 d$ & $4442.3 c-f$ & $5132 \mathrm{de}$ & $4711.1 \mathrm{~cd}$ & $4133 \mathrm{fg}$ \\
\hline ETBW7071 & $3282.6 \mathrm{~cd}$ & $3735.3 \mathrm{~cd}$ & 4060.6bcd & $4466.7 c-f$ & $5295.7 \mathrm{de}$ & $4835.9 \mathrm{bcd}$ & $4280 \mathrm{ef}$ \\
\hline ETBW7075 & $3902.5 \mathrm{a}$ & $4490 \mathrm{ab}$ & $4315.8 \mathrm{a}-\mathrm{d}$ & $4353.3 c-f$ & $6030.6 \mathrm{ab}$ & $5056.2 \mathrm{a}-\mathrm{d}$ & $4691 \mathrm{c}$ \\
\hline ETBW7076 & $4119.9 \mathrm{a}$ & $4942 \mathrm{a}$ & $4848.1 \mathrm{a}$ & $4633.5 b-e$ & $6400.2 \mathrm{a}$ & $5468.4 \mathrm{a}$ & $5069 a$ \\
\hline ETBW7077 & $3228 \mathrm{~cd}$ & $3583.5 \mathrm{~cd}$ & $4436 a-d$ & $4477.5 c-f$ & $5141.7 \mathrm{de}$ & $5152.3 \mathrm{a}-\mathrm{d}$ & $4337 \mathrm{def}$ \\
\hline ETBW7088 & $3320.3 \mathrm{~cd}$ & $3463 d$ & $4488.2 \mathrm{a}-\mathrm{d}$ & $5219.3 \mathrm{ab}$ & $5205.7 \mathrm{de}$ & 5173.1a-d & 4478 cde \\
\hline ETBW7092 & $3525 b c$ & $3668.3 \mathrm{~cd}$ & 4613.9abc & $5420 \mathrm{a}$ & $5429.4 \mathrm{~cd}$ & $5275.7 \mathrm{abc}$ & $4655 b c$ \\
\hline ETBW7104 & $3795.4 \mathrm{ab}$ & $4125.3 b c$ & $4819.6 \mathrm{a}$ & $5260.8 \mathrm{ab}$ & $5840.9 \mathrm{bc}$ & $5430.2 \mathrm{a}$ & $4880 \mathrm{~b}$ \\
\hline kingbird & $3295.3 \mathrm{~cd}$ & $3634.5 \mathrm{~cd}$ & 4361.1a-d & $4863.7 \mathrm{abc}$ & $5219.6 \mathrm{de}$ & 5066a-d & $4407 \mathrm{de}$ \\
\hline Liban & $3846.8 \mathrm{ab}$ & $3761.5 \mathrm{~cd}$ & $4663.7 \mathrm{ab}$ & $4351.8 c-f$ & $5834.7 \mathrm{bc}$ & $5572.4 \mathrm{a}$ & $4672 b c$ \\
\hline Mean & 3524.47 & 3866.71 & 4363.90 & 4581.54 & 5520.17 & 5100.16 & 4479.47 \\
\hline $\mathrm{R}^{2}(\%)$ & 82 & 77 & 54 & 67 & 85 & 55 & 88 \\
\hline $\mathrm{CV} \%$ & 5.63 & 9.24 & 9.73 & 9.49 & 4.46 & 6.87 & 8.05 \\
\hline LSD 5\% & 331.85 & 597.27 & 710.29 & 727.53 & 411.72 & 585.82 & 237.98 \\
\hline F test & $* *$ & $* *$ & $* *$ & $* *$ & $* *$ & $* *$ & $* *$ \\
\hline
\end{tabular}

$\mathrm{ETBW}=$ Ethiopia bread wheat, $\mathrm{R}^{2}=\mathrm{R}$-squire, $\mathrm{CV}=$ coefficient of variation, $\mathrm{LSD}=$ least significant different 
Table 4. Combined mean grain yield and other agronomic traits of bread wheat genotypes.

\begin{tabular}{|c|c|c|c|c|c|c|}
\hline Genotypes & DH & DM & PH & SL & SW & STPS \\
\hline ETBW7052 (G13) & $80.2 \mathrm{a}$ & $121.3 \mathrm{c}$ & $78.1 \mathrm{c}$ & $9.6 b c$ & $2.14 \mathrm{c}$ & $16.4 \mathrm{bcd}$ \\
\hline ETBW7076 (G6) & $77.8 \mathrm{~b}$ & $118.2 \mathrm{ef}$ & $75.04 \mathrm{de}$ & $10.4 \mathrm{a}$ & $3.7 \mathrm{a}$ & $19.02 \mathrm{a}$ \\
\hline ETBW7092 (G11) & $76.1 \mathrm{c}$ & $119.5 \mathrm{de}$ & $81.9 \mathrm{~b}$ & 7.9hi & $1.69 \mathrm{de}$ & $15.01 d$ \\
\hline ETBW7071 (G15) & $75.9 \mathrm{~cd}$ & $118.7 \mathrm{e}^{2}$ & $73.54 \mathrm{ef}$ & $8.8 \mathrm{ef}$ & $1.9 \mathrm{~cd}$ & $16.26 \mathrm{~cd}$ \\
\hline ETBW7072 (G8) & $75 \mathrm{de}$ & $120.5 \mathrm{~cd}$ & $76.19 \mathrm{~cd}$ & $8.3 \mathrm{gh}$ & $1.99 \mathrm{~cd}$ & $15.73 \mathrm{~cd}$ \\
\hline Liban (G9) & $74.8 \mathrm{e}$ & $121.3 \mathrm{c}$ & $74.05 \mathrm{def}$ & $8.4 \mathrm{fg}$ & $2.12 \mathrm{c}$ & $16.69 b c$ \\
\hline ETBW7104 (G3) & $71.6 \mathrm{~g}$ & $113.4 \mathrm{~h}$ & $75.3 \mathrm{de}$ & $9.2 \mathrm{~d}$ & $3.02 b$ & $17.85 \mathrm{ab}$ \\
\hline ETBW7077 (G7) & $71.4 \mathrm{~g}$ & $126.5 \mathrm{a}$ & $73.47 \mathrm{ef}$ & $9.1 \mathrm{de}$ & $2.03 \mathrm{c}$ & $15.90 \mathrm{~cd}$ \\
\hline ETBW7068 (G5) & $70.9 \mathrm{~g}$ & $114.2 \mathrm{~h}$ & $78.18 \mathrm{c}$ & $9.8 \mathrm{~b}$ & $2.02 \mathrm{c}$ & $15.05 \mathrm{~d}$ \\
\hline ETBW7056 (G2) & $70.3 i$ & $116.3 \mathrm{~g}$ & $76.48 \mathrm{~cd}$ & $9.8 \mathrm{~b}$ & $2.06 \mathrm{c}$ & $16.29 \mathrm{~cd}$ \\
\hline ETBW7075 (G10) & $69.7 \mathrm{i}$ & $114.1 \mathrm{~h}$ & $71.8 \mathrm{f}$ & $9.4 \mathrm{~cd}$ & $1.98 \mathrm{~cd}$ & $15.58 \mathrm{~cd}$ \\
\hline ETBW7088 (G14) & $69.6 \mathrm{i}$ & $114.5 \mathrm{~h}$ & $68.46 \mathrm{~g}$ & $9.2 \mathrm{~cd}$ & $1.87 \mathrm{cde}$ & $15.52 \mathrm{~cd}$ \\
\hline kingbird (G4) & $65.3 \mathrm{j}$ & $109.6 \mathrm{i}$ & $72.04 f$ & 7.9hi & $1.6 \mathrm{ef}$ & $14.98 \mathrm{~d}$ \\
\hline Mean & 73.13 & 117.89 & 76.12 & 8.92 & 2.09 & 15.9 \\
\hline $\mathrm{R}^{20} \%$ & 96 & 97 & 90 & 92 & 0.8 & 0.69 \\
\hline LSD $5 \%$ & 0.9 & 1.43 & 2.62 & 0.39 & 0.32 & 1.53 \\
\hline F test & $* *$ & $* *$ & $* *$ & $* *$ & $* *$ & $* *$ \\
\hline
\end{tabular}

Table 4. Continued.

\begin{tabular}{|c|c|c|c|c|c|}
\hline Genotypes & GPS & GPST & TSW & Kgha & YAD \\
\hline ETBW7052 (G13) & $43.09 \mathrm{bc}$ & $2.64 b-f$ & $24.37 \mathrm{~cd}$ & $4280 \mathrm{ef}$ & -8.4 \\
\hline ETBW7076 (G6) & $55.51 \mathrm{a}$ & $2.97 \mathrm{ab}$ & $36.54 \mathrm{ab}$ & $5669 a$ & 21.3 \\
\hline ETBW7092 (G11) & $33.82 f$ & $2.29 \mathrm{fgh}$ & $36.83 a$ & $4655 b c$ & -0.4 \\
\hline ETBW7069 (G12) & $37.05 \mathrm{def}$ & $2.48 \mathrm{c}-\mathrm{h}$ & $25.48 \mathrm{bcd}$ & $4133 \mathrm{fg}$ & -11.5 \\
\hline ETBW7071 (G15) & $40.48 \mathrm{~cd}$ & $2.46 \mathrm{c}-\mathrm{h}$ & $25.56 \mathrm{bcd}$ & $4280 \mathrm{ef}$ & -8.4 \\
\hline ETBW7072 (G8) & $37.08 \mathrm{def}$ & $2.41 \mathrm{~d}-\mathrm{h}$ & $25.61 \mathrm{a}-\mathrm{d}$ & $3940 \mathrm{~g}$ & -15.7 \\
\hline Liban (G9) & $45.75 b$ & $2.78 \mathrm{a}-\mathrm{d}$ & $22.64 \mathrm{~cd}$ & $4672 b c$ & 0 \\
\hline ETBW7104 (G3) & $39.75 \mathrm{cde}$ & $2.21 \mathrm{gh}$ & $33.13 \mathrm{abc}$ & $5179 b$ & 10.9 \\
\hline ETBW7077 (G7) & $33.59 f$ & $2.12 \mathrm{~h}$ & $22.06 \mathrm{~cd}$ & 4337def & -7.2 \\
\hline ETBW7068 (G5) & $35.44 \mathrm{ef}$ & $2.39 \mathrm{e}-\mathrm{h}$ & $29.60 \mathrm{a}-\mathrm{d}$ & $4568 \mathrm{~cd}$ & -2.2 \\
\hline ETBW7056 (G2) & $41.21 \mathrm{bcd}$ & $2.52 \mathrm{c}-\mathrm{g}$ & $27.04 \mathrm{a}-\mathrm{d}$ & $4877 \mathrm{c}$ & 4.4 \\
\hline ETBW7075 (G10) & $37.62 \mathrm{def}$ & $2.45 c-h$ & $28.28 \mathrm{a}-\mathrm{d}$ & $4691 \mathrm{c}$ & 0.4 \\
\hline ETBW7088 (G14) & $41.63 \mathrm{bcd}$ & $2.81 \mathrm{abc}$ & $22.54 \mathrm{~cd}$ & 4478 cde & -4.2 \\
\hline kingbird (G4) & $43.90 \mathrm{bc}$ & $3.06 \mathrm{a}$ & $21.18 \mathrm{~d}$ & $4407 \mathrm{de}$ & -5.7 \\
\hline $\mathrm{R}^{2} \%$ & 0.73 & 0.55 & 0.43 & 88 & \\
\hline LSD $5 \%$ & 5.01 & 0.37 & 11.22 & 237.98 & \\
\hline $\mathrm{F}$ test & $* *$ & $* *$ & $* *$ & $* *$ & \\
\hline
\end{tabular}

ETBW=Ethiopia bread wheat, $\mathrm{DH}=$ Days to heading, $\mathrm{DM}=$ Days to maturity, $\mathrm{PH}=\mathrm{Plant}$ height, $\mathrm{SL}=$ spike length, $\mathrm{SW}=$ spike weight, $\mathrm{STPS}=$ spikelets per spike, GPS=grain per spike, GPST=grain per spikelet, TSW=Thousand seed weight, YLD kgha ${ }^{-1}=$ Yield in kilogram per hectare, YAD=yield advantage, $\mathrm{CV}=$ Coefficient of variation, $\mathrm{R}^{2}=\mathrm{R}$-square, $\mathrm{LSD}=$ least significant

\subsection{Major Disease Reactions}

Most genotypes evaluated had significantly low scores for their corresponding economically important disease reactions (Table5). However, some genotypes (G10) and (G12) were less tolerant to stem and leaf rust and septoria. Similarly, genotypes (G12, G15, G5, G2 and G10) were less tolerant to fusarium head blight (Table 5). On the other hand, genotypes (G6 and G3) were better tolerance to stem and leaf rust and fusarium head blight (Table 5).

Table 5. Combined mean of disease reactions (1-5 scale) of bread wheat genotypes evaluated in 2017-2018 main cropping season.

\begin{tabular}{|c|c|c|c|c|}
\hline Genotypes & SR & LR & SEP & FHB \\
\hline ETBW7052 (G13) & $1 \mathrm{c}$ & $1 b$ & $1.03 \mathrm{ab}$ & $1.8 \mathrm{~b}-\mathrm{e}$ \\
\hline ETBW7076 (G6) & $1.1 \mathrm{bc}$ & $1.08 \mathrm{ab}$ & $1.03 \mathrm{ab}$ & $1.9 \mathrm{~b}-\mathrm{e}$ \\
\hline ETBW7092 (G11) & $1 \mathrm{c}$ & $1 b$ & $1 b$ & $1.3 \mathrm{f}$ \\
\hline ETBW7069 (G12) & $1.13 \mathrm{ab}$ & $1.03 \mathrm{~b}$ & $1 b$ & $2.4 \mathrm{a}$ \\
\hline ETBW7071 (G15) & $1 \mathrm{c}$ & $1 b$ & $1 b$ & $2.2 \mathrm{ab}$ \\
\hline ETBW7072 (G8) & $1.03 \mathrm{c}$ & $1.06 \mathrm{ab}$ & $1 b$ & $1.8 \mathrm{~b}-\mathrm{e}$ \\
\hline Liban (G9) & $1.03 \mathrm{c}$ & $1 b$ & $1 \mathrm{~b}$ & $1.9 \mathrm{~b}-\mathrm{e}$ \\
\hline
\end{tabular}




\begin{tabular}{lllll}
\hline Genotypes & SR & LR & SEP & FHB \\
\hline Local (G1) & $1 \mathrm{c}$ & $1 \mathrm{~b}$ & $1.03 \mathrm{ab}$ & $1.8 \mathrm{cde}$ \\
ETBW7104 (G3) & $1.04 \mathrm{bc}$ & $1 \mathrm{~b}$ & $1.07 \mathrm{a}$ & $1.03 \mathrm{ab}$ \\
ETBW7077 (G7) & $1 \mathrm{c}$ & $1.08 \mathrm{ab}$ & $1.03 \mathrm{ab}$ & $1.7 \mathrm{de}$ \\
ETBW7068 (G5) & $1.13 \mathrm{ab}$ & $1.03 \mathrm{~b}$ & $1.06 \mathrm{ab}$ & $2.1 \mathrm{abc}$ \\
ETBW7056 (G2) & $1.06 \mathrm{bc}$ & $1.11 \mathrm{ab}$ & $2.4 \mathrm{a}$ \\
ETBW7075 (G10) & $1.17 \mathrm{a}$ & $1.17 \mathrm{ab}$ & $1.03 \mathrm{ab}$ & $2.0 \mathrm{bcd}$ \\
ETBW7088 (G14) & $1 \mathrm{c}$ & $1.03 \mathrm{~b}$ & $1.8 \mathrm{~b}-\mathrm{e}$ \\
kingbird (G4) & $1 \mathrm{c}$ & $1.06 \mathrm{ab}$ & $1.9 \mathrm{~b}-\mathrm{e}$ \\
Mean & 1.04 & 1.04 & 1.02 & 1.91 \\
CV\% & 14.14 & 17.29 & 9.25 & 30.15 \\
R $\%$ & 42 & 41 & 38 & 71 \\
LSD 5\% & 0.097 & 0.12 & 0.062 & 0.38 \\
F test & $* *$ & $* *$ & $* *$ & $* *$ \\
\hline
\end{tabular}

ETBW=Ethiopia bread wheat, $\mathrm{CV}=$ Coefficient of variation, $\mathrm{LSD}=$ least significant difference, $\mathrm{R}^{2}=\mathrm{R}-\mathrm{Square}, \mathrm{SR}=$ stem rust, $\mathrm{LR}=\mathrm{leaf}$ rust, $\mathrm{SEP}=\mathrm{septoria}$, FHB=fusarium head blight. 1-5 scale where $1=$ resistant, $5=$ susceptible

\subsection{Additive Main Effects and Multiplicative Interaction (AMMI) Model}

The combined ANOVA and AMMI analysis for grain yield at six environments exhibited by bread wheat grain yield (Table 6), was significantly affected by environments. This explained $65.06 \%$ of the total treatment variation, while the $\mathrm{G}$ and GEI were significant and accounted for 13.34 and 9.44\%, respectively (Table 6). Similar findings have been reported in previous studies $[6,11]$. A study by Gauch and Zobel [9], reported in standard multi-environment trials (METs), environment effect contributes $80 \%$ of the total sum of treatments and $10 \%$ effect of genotype and interaction. In additive variance, the portioning of GEss data matrix using AMMI analysis, indicated the first PCAs were significant ( $\mathrm{P}$ $<0.01)$. PCA 1 and 2 accounted for 7.88 and $1.15 \%$ of the GE interaction, respectively; representing a total of $9.03 \%$ of the interaction variation (Table 6). Similar results have been reported in earlier studies [13].

Large yield variation explained by environments indicated that environments were diverse, with large differences between environmental means contributing maximum of the variation in grain yield (Table 7). Grain yield of environments ranged from $3524 \mathrm{kgha}^{-1}$ in E3 to $5520 \mathrm{~kg} \mathrm{ha}^{-1}$ in E2. Genotype mean grain yield varied from $3940 \mathrm{~kg} \mathrm{ha}^{-1}$ for (G8) to $5069 \mathrm{~kg} \mathrm{ha}^{-1}$ in (G6), with the over all mean of $4493 \mathrm{~kg} \mathrm{ha}^{-1}$ (Table 7).

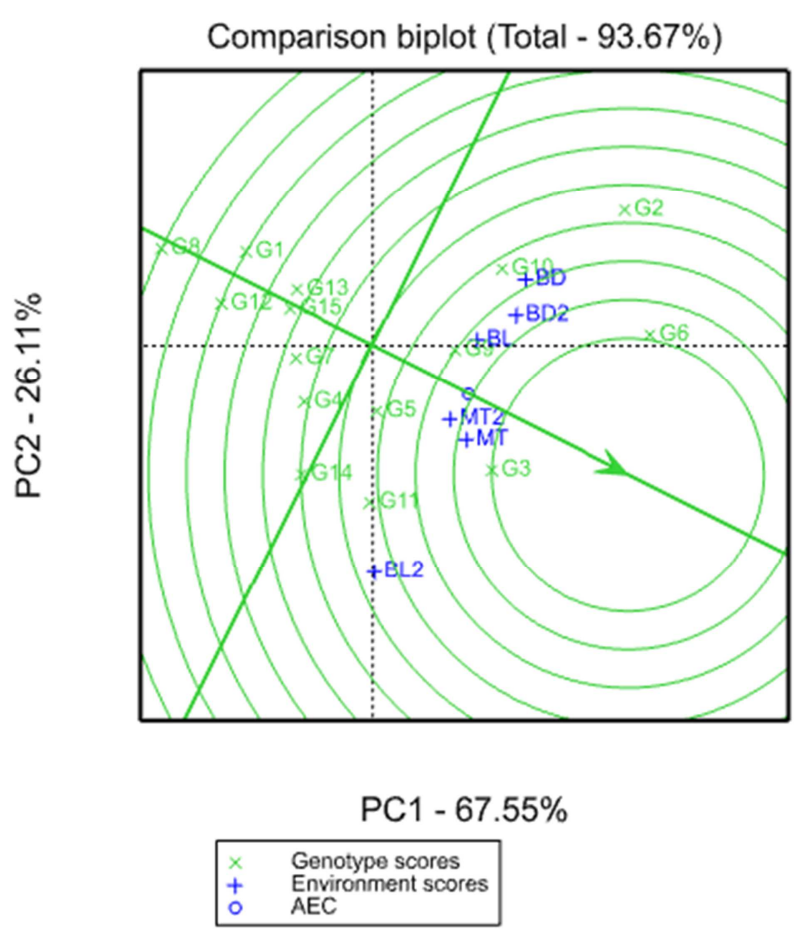

Figure 1. GGE bi-plot based on genotype-focused scaling for comparison of genotypes for their yield potential and stability of bread wheat varieties at Western Oromia in Ethiopia.

Table 6. Additive main effect and multiplicative interaction analysis of variances (AMMI) for grain yield of 15 bread wheat genotypes evaluated at six environments.

\begin{tabular}{lllll}
\hline Source of variation & DF & SS & EX. SS\% & MS \\
\hline Total & 269 & 192196683 & 100 & 714486 \\
Treatments & 89 & 168813778 & 87.83 & $1896784^{* *}$ \\
Genotypes & 14 & 25637440 & 13.34 & $1831246^{* *}$ \\
Environments & 5 & 125035456 & 65.06 & $25007091^{* *}$ \\
Block & 12 & 3230047 & 1.68 & $269171^{*}$ \\
GxE & 70 & 18140883 & 9.44 & $259155^{* *}$ \\
IPCA 1 & 18 & 15142869 & 7.88 & $841271^{* *}$ \\
IPCA 2 & 16 & 2201589 & 1.15 & $137599^{\text {ns }}$ \\
Residuals & 36 & 796424 & 0.41 & 22123 \\
Error & 168 & 20152857 & & 119957 \\
\hline
\end{tabular}

$\mathrm{DF}=$ degree of freedom, $\mathrm{SS}=$ sum of squares, MS=mean squares, IPCA=Interaction Principal Component Axis, EX. SS\%=Explained Sum of square ns $* * *$ non-Significant, Significant at the $0.5 \%$ and $0.1 \%$ level of probability, respectively 
Table 7. Average grain yield $\left(\mathrm{kg} \mathrm{ha}^{-1}\right)$ of 15 bread wheat genotypes tested across six environments in 2017-2018 main cropping seasons.

\begin{tabular}{llllllll}
\hline Gen/Env & E1 & E2 & E3 & E4 & E5 & E6 & Mean \\
\hline Local (G1) & 3582 & 5209 & 3183 & 4112 & 3966 & 4701 & 4125 \\
ETBW7075 (G10) & 4532 & 6007 & 3856 & 4354 & 4340 & 5060 & 4691 \\
ETBW7092 (G11) & 3676 & 5398 & 3555 & 5417 & 4576 & 5310 & 4655 \\
ETBW7069 (G12) & 3485 & 5108 & 3140 & 4447 & 3946 & 4674 & 4133 \\
ETBW7052 (G13) & 3843 & 5370 & 3340 & 4480 & 3965 & 4683 & 4280 \\
ETBW7088 (G14) & 3442 & 5200 & 3369 & 5217 & 4452 & 5190 & 4478 \\
ETBW7071 (G15) & 3707 & 5312 & 3313 & 4465 & 4076 & 4804 & 4279 \\
ETBW7056 (G2) & 4836 & 6381 & 4129 & 3829 & 4674 & 5416 & 4877 \\
ETBW7104 (G3) & 4126 & 5795 & 3859 & 5256 & 4752 & 5485 & 4879 \\
kingbird (G4) & 3564 & 5268 & 3362 & 4864 & 4323 & 5060 & 4407 \\
ETBW7068 (G5) & 3688 & 5458 & 3537 & 4791 & 4595 & 5344 & 4569 \\
ETBW7076 (G6) & 4885 & 6393 & 4237 & 4628 & 4771 & 5497 & 5069 \\
ETBW7077 (G7) & 3464 & 5244 & 3313 & 4480 & 4383 & 5135 & 4337 \\
ETBW7072 (G8) & 3256 & 4941 & 2960 & 4034 & 3856 & 4596 & 3940 \\
Liban (G9) & 3915 & 5720 & 3713 & 4350 & 4785 & 5548 & 4672 \\
Mean & 3867 & 5520 & 3524 & 4582 & 4364 & 5100 & 4493 \\
\hline
\end{tabular}

Gen=genotype; Env=environment, E1-BD-2017 (Bedesso), E2-BD-2018, E3-BL-2017 (Belem), E4-BL-2018, E5-MT-2017 (Mata), E6-MT-2018, the number following each location indicates the year, $\mathrm{E}=$ environment

The average environment is defined by the average values of $\mathrm{PC} 1$ and 2 for the all environments, and it is presented with a circle [14]. The average ordinate environment (AOE) is defined by the line which is perpendicular to the AEA (average environment axis) line and pass through the origin. This line divides the genotypes in to those with higher yield than average and in to those lower yield than average. By projecting the genotypes on AEA axis, the genotypes are ranked by yield; where the yield increases in the direction of arrow. In this case, the highest yield had genotypes G6, G3 and G2, but the lowers had G8, G1 and G12 (Figure 1). Stability of the genotypes depends on their distance from the $\mathrm{AE}$ abscissa. Genotypes closer to or around the center of concentric circle indicated these genotypes are more stable than others. Therfore, the greatest stability in the high yielding group had genotypes G6, G3 and G2, whereas the most stable and yielder of all was G6 (Figure 1)

The genotype ranking is shown on the graph of genotype so-called "ideal" genotype (Figure 1). An ideal genotype is defined as one that is the highest yielding across test environments and it is completely stable in performance (that ranks the highest in all test environments; such as genotypes G6, G3 and G2 [6, 19], Even though such an "ideal" genotype may not exist in reality, it could be used as a reference for genotype evaluation [12].

A genotype is more appropriate if it is located closer to "ideal" genotype [6, 11]. So, the closer to the "ideal" genotype in this study was G6 (Figure 1). The ideal test environment should have large PC1 scores (more power to discriminate genotypes in terms of the genotypic main effect) and small (absolute) PC2 scores (more representative of the overall environments). Such an ideal environment was represented by an arrow pointing to it (Figure 2). Actually, such an ideal environment may not exist, but it can be used as an indication for genotype selection in the METs.

An environment is more desirable if it is located closer to the ideal environment. Therefore, using the ideal environment as the center, concentric circles were drawn to help visualize the distance between each environment and the ideal environment [22]. Accordingly, E3 (BL-2017=Belem), which fell into the center of concentric circles, was an ideal test environment in terms of being the most representative of the overall environments and the most powerful to discriminate genotypes (Figure 2).

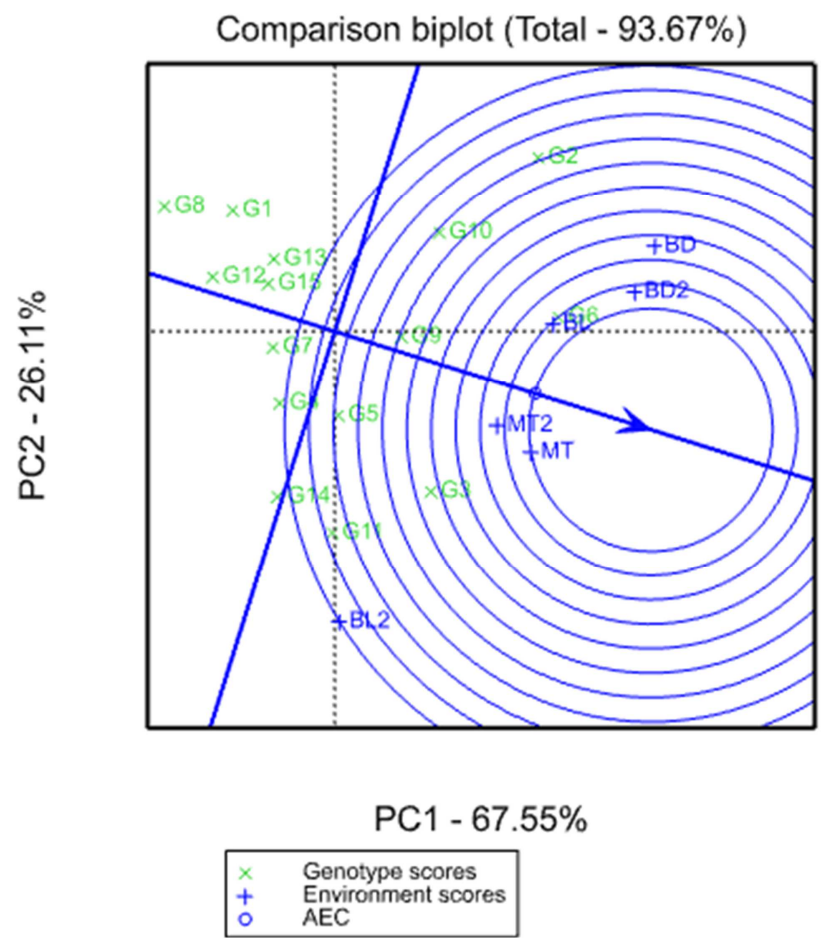

Figure 2. GGE bi-plot based on tested environments-focused comparison for their relationship E1-BD-2017 (Bedesso), E2-BD-2018, E3-BL-2017 (Belem), E4-BL-2018, E5-MT-2017 (Mata), E6-MT-2018, the number following each location indicates the year, E=environment. 


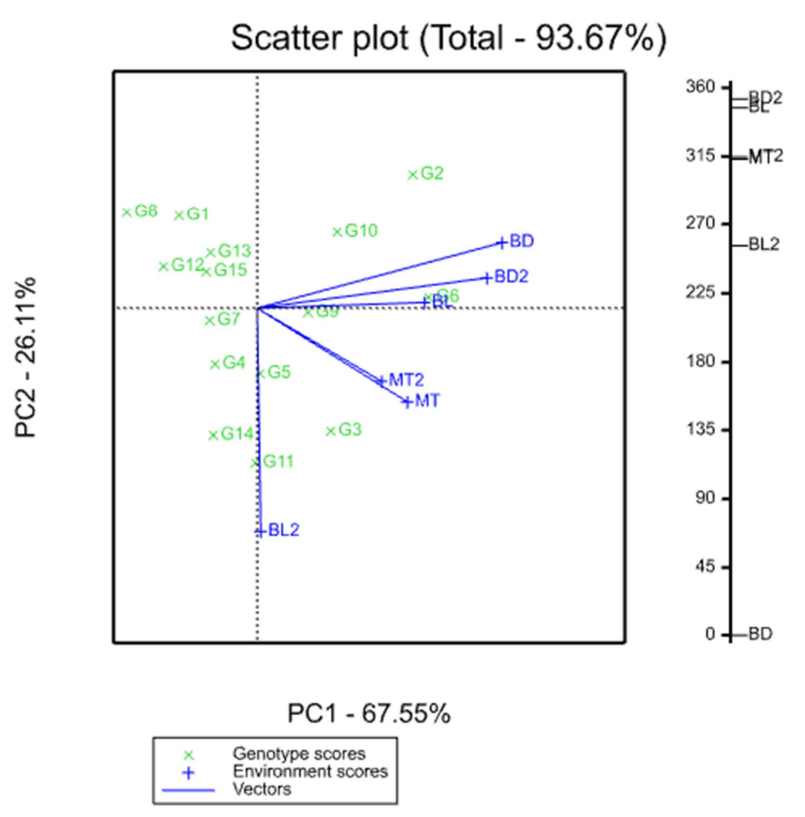

Figure 3. GGE bi-plot based on tested environments-focused comparison for their relationships. E1-BD (Bedesso), E2-BD-2018, E3-BL-2017 (Belem), E4-BL-2018, E5-MT-2017 (Mata), E6-MT-2018, the number following each location indicates the year, $E=$ environment.

The correlation coefficients among the six test environments and the vector view of the GGE-bi-plot delivered a brief summary of the interrelationship between the environments and correlation coefficients were significant (Figure 3). Most environments were positively correlated since the angles among them were smaller than $90^{\circ}$, apart from environment E4 (Belem-2018), which had negatively correlated with E1 (BD-Bedesso-2017) and E2 (BD-Bedesso-2018) since obtuse angles between them (Figure 3). Similarly, Farshadfar et al. [6], reported environments ER3 and EI3 which represented rain fed and irrigated conditions in 2011 cropping seasons, respectively, made an obtuse angle with each other, indicated a negative correlation between the response of genotypes to rain fed and irrigated conditions. Indirect selection could be functional in the case where the same character was measured on the same genotypes in different environments. Where there are no correlations of error effects among environments, the phenotypic correlation between environments may be used to investigate indirect response to selection [3]. Indirect selection for grain yield can be partial across the tested environments. This means, for instance, the genotypes adaptable or higher productivity in E4 may also show similar responses to E5 and E6 as well.

Table 8. Correlation coefficients among six test environments.

\begin{tabular}{llllll}
\hline Environment & E1 & E2 & E3 & E4 & E5 \\
\hline E2 & $0.984^{* *}$ & & & & \\
E3 & $0.936^{* *}$ & $0.977^{* *}$ & & & \\
E4 & $0.197^{\mathrm{ns}}$ & $-0.134^{\mathrm{ns}}$ & $0.066^{\mathrm{ns}}$ & & \\
E5 & $0.571^{*}$ & $0.701^{* *}$ & $0.82^{* *}$ & $0.387^{\mathrm{ns}}$ & \\
E6 & $0.554^{*}$ & $0.687^{*}$ & $0.806^{* *}$ & $0.378^{\mathrm{ns}}$ & $0.999 * *$ \\
\hline
\end{tabular}

ns, $* * *$ non- Significant, significant at the 0.05 and 0.01 probability level, respectively.

\subsection{Additive Main Effects and Multiple Interactions (AMMI)}

\subsubsection{AMMI Stability Value (ASV)}

Genotypes exhibited significant genotype by environment interaction effects and the additive and multiplicative interaction effect stability analysis (ASV) implied splitting the interaction effect. In view of the mean grain yield as a first criterion for evaluating, G6 was the highest mean grain yield $\left(5069 \mathrm{kgha}^{-1}\right)$, followed by the genotypes G3 and G2 with the mean grain yield of (4879 and $4877 \mathrm{kgha}^{-1}$, respectively). Whereas, genotypes G8, G12 and G1 were with low mean grain yields across the testing locations (Table 9). The IPCA1 and 2 scores in the AMMI model are indicators of stability [14]. Considering IPCA1, G6 was the most stable genotype with IPCA1 value (-16.65), followed by G3 with IPCA1 value of (6.95). Likewise, in IPCA2, G9 was the most stable with interaction principal component value (18.03). The two principal components have their own extremes; however, calculating the AMMI stability value (ASV) is a balanced measure of stability [14]. Genotypes with lower ASV values are considered more stable and genotypes with higher ASV are unstable. According to the ASV ranking in the (Table 9), G8 was the most stable with an ASV value of 1 followed by G1 with

Table 9. AMMI stability value, AMMI rank, yield, yield rank and genotype selection index and principal component axis.

\begin{tabular}{|c|c|c|c|c|c|c|c|}
\hline Genotypes & ASV & ASV rank & YLD & YLD rank & GSI & IPCAg1 & IPCAg2 \\
\hline G6 & 144.3 & 10.0 & 5069 & 1.0 & 11.0 & -16.65 & 5.04 \\
\hline G3 & 61.4 & 5.0 & 4879 & 2.0 & 7.0 & 6.95 & 2.55 \\
\hline G2 & 245.6 & 15.0 & 4877 & 3.0 & 18.0 & -28.98 & -6.11 \\
\hline G10 & 148.8 & 12.0 & 4691 & 4.0 & 16.0 & -15.43 & 9.17 \\
\hline G9 & 153.4 & 14.0 & 4672 & 5.0 & 19.0 & -4.16 & -18.03 \\
\hline G11 & 145.9 & 11.0 & 4655 & 6.0 & 17.0 & 17.29 & 3.27 \\
\hline G5 & 86.4 & 7.0 & 4569 & 7.0 & 14.0 & 6.82 & -7.88 \\
\hline G4 & 82.2 & 6.0 & 4407 & 9.0 & 15.0 & 9.88 & 0.72 \\
\hline G7 & 92.9 & 8.0 & 4337 & 10.0 & 18.0 & 5.42 & -9.80 \\
\hline G13 & 100.3 & 9.0 & 4280 & 11.0 & 20.0 & -1.71 & 11.97 \\
\hline G15 & 44.1 & 3.0 & 4279 & 12.0 & 15.0 & 0.58 & 5.29 \\
\hline G12 & 56.6 & 4.0 & 4133 & 13.0 & 17.0 & 4.02 & 5.51 \\
\hline G1 & 27.2 & 2.0 & 4125 & 14.0 & 16.0 & -3.16 & 0.86 \\
\hline G8 & 23.2 & 1.0 & 3940 & 15.0 & 16.0 & 1.16 & -2.54 \\
\hline
\end{tabular}


ASV value 2. However, G2 was the most unstable since higher ASV value of 15. The stable genotype was followed with mean grain yield above the grand mean and this result was in agreement with Hintsa and Abay [10], who has used ASV as one method of evaluating grain yield stability of bread wheat varieties in Tigray and similar reports been made by Abay and Bjørnstad [1]; Sivapalan et al. [16] in barley in Tigray and bread wheat using AMMI stability value. A genotype with the least of genotype selection index (GSI) is considered as the most stable genotype [5]. Accordingly, G6 was the most stable genotype since with the low of genotype selection index (GSI) and the highest mean grain yield of all (Table 9).

\section{Conclusion}

Based on the two analyses of AMMI and GGE-bi-plot models, G6 and G3 considered by high yield and more stability, consequently, G6 close to ideal genotype, so this genotype is adaptable to a wide range of environmental conditions. Therefore, G6 was identified as ideal genotypes in terms of yielding ability and stability, tolerant to diseases for advancement, release and use as parents in future breeding programs.

\section{Acknowledgements}

The authors greatly acknowledged Oromia Agricultural Research Institute (IQQO) for financial support. And Kulumsa Agricultural Research Center is acknowledged for the provision of test materials.

\section{References}

[1] Abay, F. and Bjørnstad, A. 2009. Specific adaptation of barley varieties in different locations in Ethiopia. Euphotic 167: 181195.

[2] Comstock, R. E. and Moll, R. H. 1963. Genotype x Environment Interactions: Symposium on Statistical Genetics and Plant Breeding. National Academy Science National Research Council, Washington, D. C. pp. 164-196.

[3] Cooper, M. and Delacy, I. H. 1994. Relationships among analytic methods used to study genotypic variation and genotype-by-environment interaction in plant breeding multienvironment trials. Theory Applied Genetic 88: 561-572.

[4] Delacy, I. H., Basford, K. E., Cooper, M. and Bull, J. K. 1996. Analysis of multi- environment trials- an historical perspective. Plant Adaptation and Crop Improvement. Cooper, M. and Hammer, G. L. (Eds.). CAB International. pp. 39-124.

[5] Farshadfar, E. 2008. Incorporation of AMMI stability value and grain yield in a single non-parametric index (Genotype Selection Index) in bread wheat. Pakistan Journal of Biological Sciences11: 1791-1796.

[6] Farshadfar, E., Mohammadi, R., Aghaee, M. andVaisi, Z. 2012 GGE biplot analysis of genotype $\times$ environment interaction in wheat-barley disomic addition lines. Australia Journal of Crop Sciences 6: 1074-1079.
[7] Gabriel, K. R. 1971. The biplot graphic of matrices with application to principal component analysis Biometrics 58: 453-467.

[8] Gauch, H. G. 2006. Statistical analysis of yield trials by AMMI and GGE Crop Sciences 46: 1488-1500.

[9] Gauch, H. G. and Zobel, R. W. 1997. Interpreting megaenvironments and targeting genotypes. Crop Sciences 37: 311326.

[10] Hintsa, G. and Abay, F. 2013. Evaluation of bread wheat genotypes for their adaptability in wheat growing areas of Tigray Region, northern Ethiopia. Journal of Biodiversity and Endangered Species.

[11] Kaya, Y., Akcura, M. andTaner, S. 2006. GGE-bi-plot analysis of multi- environment yield trials in bread wheat. Turkish Journal of Agriculture 30: 325-337.

[12] Mitrovic, B., Stanisavljevi, D., Treski, S., Stojakovic, M., Ivanovic, M., Bekavac, G. and Rajkovic, M. 2012. Evaluation of experimental Maize hybrids tested in Multi-location trials using AMMI and GGE bi-plot analysis. Turkish Journal of Field Crops 17: 35-40.

[13] Mohammadi, R. and Amri, A. 2009. Analysis of genotype $\times$ environment interactions for grain yield in durum wheat. Crop Sciences 49: 1177-1186.

[14] Purchase, J. L. 1997. Parametric analysis to describe genotype $\mathrm{x}$ environment interaction and yield stability in winter wheat Ph. D. Thesis, Department of Agronomy, Faculty of Agriculture of the University of the Free State, Bloemfontein, South Africa.

[15] SAS Institute Inc. 2008. Statistical analysis Software version 9.2, Cary, NC: SAS Institute Inc. USA.

[16] Sivapalan, S., Obrien, L., Ortiz-Ferrara, G., Hollamby, GJ., Barclay, I. and Martin, PJ. 2000. An adaptation analysis of Australian and CIMMYT/ICARDA wheat germplasm in Australian production environments. Crop Science Pastures 51: 903-915.

[17] VSN International, 2012. "Gen Stat for Windows 15th Edition. VSN International, Hemel Hempstead, UK. Available: www.genStat.co.uk.

[18] Yan, W. 2001. GGE bi-plot- a windows application for graphical analysis of multi-environment trial data and other types of two-way data Journal of Agronomy93: 1111-1118.

[19] Yan, W. and Kang, MS. 2003. GGE bi-plot analysis: a graphical tool for breeders, In: Kang MS. (Ed). Geneticists, and Agronomist. CRC Press, Boca Raton, FL. pp. 63-88.

[20] Yan, W. and Hunt, L. A., Sheng, Q. and Szlavnics, Z. 2000. Cultivar evaluation and mega environment investigation based on the GGE bi-plot. Crop Science 40: 597-605.

[21] Yan, W. and Hunt, LA. 2001. Genetic and environmental causes of genotype by environment interaction for winter wheat yield in Ontario. Crop Science 41: 19-25.

[22] Yan, W. and Rajcan, I. 2002. Bi-plot analysis of test sites and trait relations of soybeanin Ontario. Crop Science 42: 11-20. 